Rok XVI (2021) | 1 (31) | S. 247-252

https://doi.org/10.12797/LV.16.2021.31.19

Licencja: CC BY-NC-ND 4.0

Fabian Kaulfürst $\bullet$

Serbski institut, Chóśebuz

fabian.kaulfuerst@serbski-institut.de

\title{
WITOLD TASZYCKI UND DIE SORABISTIK
}

Słowa klucze: Witold Taszycki, sorabistyka, Serbowie Łużyccy, stosunki polsko-serbołużyckie Stichwort: Witold Taszycki, Sorabistik, Sorben, polnisch-sorbische Beziehungen

Keywords: Witold Taszycki, Sorbian Studies, Sorbs of Lusatia, Polish-Sorbian relations

Josef Páta, einer der bekanntesten tschechischen Sorabisten seiner Zeit, bezeichnete Witold Taszycki im „Česko-lužický věstník“ (Páta 1926: 20) als einen der besten polnischen Kenner der sorbischen Problematik. Sein sorabistisches Werk wurde bisher mehrmals gewürdigt. Jan Petr (Petr 1958, 1968) verfasste zwei Aufsätze anlässlich des 60. und 70. Geburtstags Taszyckis, Edward Zych (1969) stellte ihn als bedeutenden Freund der Sorben vor und Hinc Šewc (Šewc 1980) sowie Jerzy Rusek (1981) beleuchteten sein sorabistisches Schaffen nach seinem Ableben.

Der Name Witold Taszycki taucht im sorabistischen Kontext wohl 1916 erstmals auf: In den Protokollauszügen des sorbischen wissenschaftlichen Vereins Maćica Serbska findet sich die schlichte Notiz: „Nowy sobustaw Witołd Taszycki w Podgorze-Krakowje so přija“" (ČMS: 117). Taszycki hatte sich bereits 1915 als Gymnasiast um die Mitgliedschaft bemüht (vgl. Petr 1968: 5). Sein Interesse für die Sorben und die Sorabistik fand volle Unterstützung durch die Professoren Jan Rozwadowski und Kazimierz Nitsch, beide seit 1900 ebenfalls Mitglieder der Maćica Serbska (vgl. ibid.). Später konnte gemeinsam mit Vilim Frančić und Kazimierz Piekarski in Krakau sogar eine eigene Maćica-Gruppe gegründet werden, die Muka (1923²: 19) „Krakowska škupina [sic!] abo župa“ nennt, und deren Sekretär Witold Taszycki war.

1 Deutsch: Aufgenommen wurde ein neues Mitglied, Witold Taszycki in Podgórze-Krakau.

2 Petr (1968: 5) gibt fälschlich 1924 an. 
Bei seinem ersten Besuch im Jahre 1922 weilte Taszycki von Juli bis Oktober in der Lausitz. Dabei machte er die Bekanntschaft namhafter sorbischer Persönlichkeiten. Petr (1968: 6) nennt: Arnošt Muka, Marko Smoler, Ota Wićaz, Bjarnat Krawc, Jan Skala, Jan Bryl, Michał Nawka, Marja Kubašec, Józef Nowak, Mina Witkojc, Bogumił Šwjela, Měrćin Nowak-Njechorński, Herman Šleca. Als Begleiter Taszyckis bzw. als dessen Führer durch die Lausitz nennt Petr (ibid.) Michał Wjerab. An gleicher Stelle weist Petr darauf hin, dass Taszycki bei seinem Lausitzaufenthalt auch einflussreiche tschechische Freunde der Sorben kennen lernt, nämlich Ludvík Kuba, Josef Páta, Jan Hejret und Vladimír Zmeškal; Adolf Černý hatte er im selben Jahr bereits zuvor in Warschau getroffen. Die Kontakte zu diesen tschechischen Aktivisten vertiefte Taszycki später während eines längeren Aufenthalts in Prag (ibid.).

Der zweite Lausitzbesuch Taszyckis erfolgte im Juli/August 1923 und war vor allem dem Studium sorbischer Sprachdenkmäler des 16. Jahrhunderts gewidmet. Petr (ibid.) nennt den handschriftlichen Wolfenbütteler Psalter sowie das Gesangbuch und den Katechismus des Albinus Mollerus. Aufgrund der allgemein unruhigen politischen Lage musste Taszycki die Lausitz vorzeitig verlassen. Er plante zwar weitere Reisen in die Lausitz, musste jedoch davon Abstand nehmen. Unter anderem warnte ihn sein ehemaliger Begleiter Wjerab 1924 (oder 1925) vor der Bautzener Polizei und wurde selbst deswegen verhört (vgl. Petr 1968: 6).

Innerhalb seines umfangreichen slawistischen Werks hat das sorabistische Schaffen Taszyckis zwar nicht den größten Umfang, kann aber nicht als marginal abgetan werden. Petr (ibid.: 10 f.) zählt in seiner „Bibliografija sorabikow prof. dr. W. Taszyckeho" nicht weniger als 33 Positionen, manche davon wurden mehrfach veröffentlicht. Der Stellenwert der Sorabistik für Taszycki wird deutlich, wenn man sich vor Augen führt, dass er sich im Rahmen seiner Habilitation mit der Stellung des Sorbischen unter den westslawischen Sprachen auseinandersetzte. $\mathrm{Zu}$ diesem Thema hielt er am 27. Oktober 1925 einen Vortrag (ibid.: 4), der 1928 im Druck erschien (Taszycki 1928). Der im gegebenen Kontext als Pionierarbeit zu bezeichnende Aufsatz mit dem Namen Stanowisko języka łużyckiego ist zweifellos der bedeutendste sprachwissenschaftlich orientierte sorabistische Beitrag Taszyckis. In ihm stellt Taszycki einführend damals konkurrierende Aussagen verschiedener Autoren zur Stellung des Sorbischen innerhalb des Westslawischen vor. Er präsentiert u. a. die Meinung August Schleichers, das Sorbische wäre Teil der tschechischen Untergruppe, die Position Baudouin de Courtenays, dass innerhalb des Westslawischen eher das Lechische und das Sorbische eine gemeinsame Untergruppe bilden sowie die Aussage von Ramułt und Lorentz, dass das Sorbische eine eigenständige Untergruppe darstellt. Er hebt die Arbeiten der Mitglieder seiner Krakauer Maćica-Gruppe hervor: Nitsch weist Schleichers These zurück und verortet das Sorbische in einer Zwischenstellung zwischen der lechischen und der tschechischen Gruppe, Rozwadowski teilt das Westslawische in eine lechische, eine sorbische und eine tschechisch-slowakische Gruppe (Taszycki 1928: 127 f.). Kritisch erwähnt Taszycki (ibid.: 128) einen Aufsatz 
Šachmatovs, in welchem dieser 29 sorbische historisch-phonetische Spezifika herausgearbeitet hatte. Nach dieser Bestandsaufnahme versucht Taszycki zu zeigen, dass das Lechische und Sorbische ursprünglich eine Untergruppe des Westslawischen bildeten. Dafür zählt er zuerst die ihm wichtig erscheinenden gemeinsamen Entwicklungen des Lechischen und Sorbischen auf (ibid.: 128-135). Danach verweist er auf einige tschechisch-sorbische Gemeinsamkeiten (ibid.: 135-137) und wendet sich zum Schluss Alleinstellungsmerkmalen des Sorbischen zu (ibid.: 137 f.).

$\mathrm{Zu}$ den lechisch-sorbischen Gemeinsamkeiten zählt Taszycki die Entwicklung der urslawischen Gruppen telt, tolt, tert, tort zu tlet, tlot, tret, trot, die Entwicklung der urslawischen Gruppen $t r t$, trót, $t t_{0} t, t_{0}$ 't, den Übergang von $e>o$ in verschiedenen Positionen und weitere. Bereits Wirth (1932: 225-230) kritisiert hier einige Argumentationen Taszyckis. Auffällig ist u. a., dass im Abschnitt zu den lechischsorbischen Gemeinsamkeiten auch Merkmale aufgezählt werden, die nicht nur in den lechischen und sorbischen Sprachen, sondern auch im Tschechischen auftreten (die Entwicklung der Gruppen ort, olt im Anlaut). Darauf wird zwar an der entsprechenden Stelle (Taszycki 1928: 129) hingewiesen, lässt aber den Abschnitt zu lechisch-sorbischen Gemeinsamkeiten umfangreicher erscheinen. Schwer zu erklären ist auch, warum die Entwicklung von ${ }^{*} e$ diesem Abschnitt zugeordnet wird. Es gibt zwar vereinzelte historische sorbische Texte, die in diesem Kontext Ähnlichkeiten mit der Entwicklung in den lechischen Sprachen aufweisen, diese präsentieren jedoch nicht das eigentliche Ober- bzw. Niedersorbische. Da die Entwicklung von ${ }^{{ }^{*}} e$ auch im Abschnitt zu tschechisch-sorbischen Übereinstimmungen herangezogen wird, kann man sich des Eindrucks nicht erwehren, dass auch hier versucht wird, den Abschnitt mit lechisch-sorbischen Gemeinsamkeiten künstlich zu erweitern.

Die Übereinstimmungen zwischen Tschechisch und Sorbisch haben nach Taszycki eine andere Qualität als die lechisch-sorbischen. Beispielsweise handelt es sich beim Verlust der Nasalvokale um eine allgemeine slawische Tendenz, die lediglich das Lechische nicht erreicht. Es handelt sich also nicht um eine spezifisch tschechisch-sorbische Innovation. Auch weitere - ausgewählte - Gemeinsamkeiten erweisen sich in der Analyse Taszyckis als nicht wirklich konstituierend für eine tschechisch-sorbische Untergruppe: Den Initialakzent im Tschechischen, Slowakischen und den sorbischen Sprachen weist er als Ergebnis verschiedener Ausgleichsprozesse als gemeinsames Spezifikum einer tschechisch-sorbischen Gruppe zurück. Auch die Entwicklung von $3(=\widehat{\mathrm{dz}})>\mathrm{z}$ ist für ihn kein auf das Sorbische und Tschechische beschränkter Prozess, sondern findet sich teilweise auch im Lechischen (Taszycki 1928: 137). Der für das Obersorbische und Tschechische typische Übergang von urslawischem ${ }^{*} g>h$ ist für Taszycki aus chronologischen Gründen kein Hinweis für eine nähere Verwandtschaft der beiden Sprachen.

Als einziges typisch sorbisches phonetisches Spezifikum macht Taszycki die Aussprache der Kontinuanden von urslawischem ¥ě aus. Ansonsten konstatiert er „Kiedy się jednak cofniemy w przeszłość, w dawniejsze okresy języka łużyckiego, 
zauważymy, że cechy wyróżniające łużycczyznę redukują się znacznie, dochodząc do liczby poprostu znikomej “"3 (ibid.). Diese Argumentation ist natürlich nicht zulässig, denn sie trifft allgemein auf alle slawischen (und indoeuropäischen) Sprachen zu.

So kommt Taszycki (ibid.: 138) zum Schluss, dass die westslawischen Sprachen in historischer Perspektive zu teilen sind in eine ursprünglich lechisch-sorbische und eine tschechisch-slowakische Gruppe. Diese Einschätzung wird allerdings später von Schuster-Šewc (1986 und 2013), der sich vor allem im Aufsatz von 1986 explizit mit den Thesen Taszyckis auseinandersetzt, verworfen.

Ein umfangreiches sorabistisches Projekt, das Taszycki 1922 in Angriff genommen hatte, nämlich die Edition des handschriftlichen Wolfenbütteler Psalters, kam nicht zur Ausführung. 1923 verweilte Taszycki in Wolfenbüttel und fertigte eine Abschrift des Psalters an, die sich 1968 wohl noch in seiner Bibliothek befand (vgl. Petr 1968: 8) ${ }^{4}$. Taszycki fand in den 1920er Jahren in Polen allerdings keinen Verleger und trat schließlich uneigennützig zugunsten der Edition von Reinhold Trautmann (1928) von seinem Ansinnen zurück, wofür sich Trautmann in seinem Vorwort ausdrücklich bedankt. Taszycki rezensiert schließlich die Psalter-Edition 1931 in der Zeitschrift „Ruch Słowiański“. Ein ähnliches Schicksal erlitt die von Taszycki geplante Bearbeitung von Mollers Gesangbuch und Katechismus. Nach der Abschrift von etwa 9o Blättern erfuhr Taszycki, dass Arnošt Muka bereits an einer Edition arbeitete und trat wiederum zurück (Petr 1968: 8).

Wichtig für die Sorabistik ist Taszycki auch als Rezensent. So beurteilte er unter anderem Schwelas Vergleichende Grammatik der ober- und niedersorbischen Sprache, die 1926 in Bautzen erschienen war und die 1923 in Leipzig erschienene Warichius-Edition von Meyer. Hart ins Gericht geht er mit der Wendischen (Sorbischen) Bibliographie von Jatzwauk (Leipzig 1929). Er weist auf einige in der Bibliographie fehlende (vor allem in Polen erschienene) sorabistische Schriften hin und kritisiert Fehlzuordnungen. ${ }^{5}$ Sein Fazit „To nie Bibljografja łużycka, to co najwyżej Materjały do łużyckiej bibljografji. Dodajmy: materjały niezbyt starannie zbierane“ (Taszycki 1930a: 204) ist jedoch überzogen. Jatzwauks Schrift zählt noch heute zu den sorabistischen Standardwerken auf diesem Gebiet, obgleich sie mit Sicherheit nicht fehlerlos ist. Es ist an dieser Stelle nicht möglich, auf sämtliche von Taszycki verfassten Rezensionen mit sorabistischem Hintergrund im Einzelnen einzugehen. Hervorgehoben sei, dass auch in seine allgemeinen onomastischen Arbeiten sein Wissen zur sorbischen Namenskunde mit eingeflossen ist (vgl. Petr 1968: 5).

3 Deutsch: Wenn wir jedoch in die Vergangenheit, in frühere Epochen der sorbischen Sprache zurückgehen, stellen wir fest, dass die Besonderheiten des Sorbischen deutlich reduziert sind und nur noch eine verschwindend geringe Zahl erreichen.

4 Es wäre interessant danach zu forschen, ob sie in seinem Nachlass erhalten ist.

5 Moniert wird bspw. die Zuordnung von Ćišinskis Epos Nawoženja zur Theaterliteratur. 
Neben seinen sprachwissenschaftlich orientierten Publikationen mit sorabistischem Hintergrund veröffentlichte Taszycki mehrere Informationsartikel zur sorbischen Kultur und Literatur, vor allem im Kontext der Zwischenkriegszeit. Besonders widmete er sich jedoch der Rezeption der Werke Sienkiewiczs bei den Sorben (vgl. Taszycki 193ob). Von Wert für die kulturwissenschaftliche und geschichtliche sorabistische Forschung ist seine kommentierte Edition von Briefen Jan Arnošt Smolers und Michał Hórniks an Kraszewski (Taszycki 1938).

Taszycki war aber auch als Lehrkraft an verschiedenen Universitäten ein Multiplikator für die Sorabistik. Wie Petr (1968: 9) darlegt, hielt Taszycki mehrere Vorlesungen zum Sorbischen bzw. zur sorbischen Kultur.

Mit seinem Wirken hat Taszycki auf vielfältige Art und Weise die polnisch-sorbischen, aber auch polnisch-tschechisch-sorbischen Beziehungen seiner Zeit mitgestaltet. Er unterhielt Kontakte zu zahlreichen Sorben in und außerhalb der Lausitz. Wie aus seinem Briefwechsel mit dem Ehepaar Krječmar hervorgeht (vgl. Mětšk 1972), galt sein Interesse dabei auch privaten Befindlichkeiten seiner sorbischen Bekannten.

\section{Literatur}

ČMS: „Časopis Maćicy Serbskeje“ 1916, lětnik LXIX, zešiwk I, II (cyłeho rjada čisło 134, 135). MĚTšK F., 1972, Sorabistiske listowanje Witolda Taszyckeho z Markétu Krječmarjowej a z Mikławšom Krječmarjom w Praze (1922-1931), „Pamiętnik Słowiański“ 22, S. 371-381.

Muka A., 1923, Zapiski Maćicy Serbskeje, „Časopis Maćicy Serbskeje“ 69, S. 3-54.

PÁtA J., 1926, „Česko-lužický věstník“ VII, 3, Praha.

Petr J., 1958, Profesor W. Taszycki šésćdźesatnik, „Rozhlad“ 8, 6, S. 189-193.

Petr J., 1968, Poćahi prof. dr. phil. Witolda Taszyckeho k Serbstwu. Při składnosći jeho sydomdźesatych narodninow, „Lětopis Instituta za serbski ludospyt“, rjad A, 15, 1, S. 1-11.

Rusek J., 1981, Stowiańskie zainteresowania językoznawcze i filologiczne Witolda Taszyckiego, „Poradnik Językowy“ Nr. 8-10, S. 401-408.

SCHUSTER-ŠEWC H., 1986, Stanowisko języków łużyckich w świetle nowych osiagnięć dialektologii historycznej, „Acta Universitatis Lodziensis. Folia Linguistica“ 12, S. 275-287.

SCHUSTER-ŠEWC H., 2013, Das Sorbische - Genese und sprachlicher Status, „Lětopis. Časopis za rěč, stawizny a kulturu Łužiskich Serbow" 60, 2, S. 86-94.

ŠEwC H., 1980, Witold Taszycki njebohi, „Lětopis Instituta za serbski ludospyt“, rjad A, 27, 1, S. 1.

TASZYCKI W., 1928, Stanowisko języka łużyckiego wśród języków zachodniosłowiańskich, [in:] Symbolae grammaticae in honorem Ioannis Rozwadowski, Bd. 2, Cracoviae, S. 127-138.

TAsZYCKI W., 1930a, Rec.: Jacob Jatzwauk „Wendische (Sorbische) Bibliographie“, „Ruch Słowiański“" 3-4 (3), S. 202-205.

TAszycki W., 193ob, Sienkiewicz w piśmiennictwie łużyckiem, „Ruch Słowiański“ 8-9 (3), S. 293-306.

TAszycki W., 1938, J.I. Kraszewski a Łużyczanie, „Przegląd Współczesny“ LXIV, S. 419-432. 
Trautmann R. (Hrsg.), 1928, Der Wolfenbütteler Niedersorbische Psalter, Leipzig.

Wirth P., 1932, Wendisch, [in:] Grundriß der Sächsischen Volkskunde, Leipzig, S. 225-230. ZYch E., 1969, Znamjenity prééel Serbow, „Rozhlad“19, 6, S. 221-225.

\section{Witold Taszycki and Sorbian Studies Summary}

Witold Taszycki was one of the best-known Polish experts in Sorbian studies of his time. He became enthusiastic about Sorbs at a young age. In 1916 he joined the Maćica Serbska. Twice, in 1922 and 1923, he visited Lusatia.

His most important linguistic work, explicitly devoted to Sorbian and directly related to his habilitation process, deals with the position of the Sorbian language within the West Slavic linguistic area. After analysing selected historical phonetic features, he divides this main group of Slavic into two original subgroups: Lechitic-Sorbian and Czech-Slovakian. His other major philological-linguistic works on Sorbian language remain unfinished, including the edition of the Wolfenbüttel Lower Sorbian Psalter and the hymnal and the catechism of Albinus Mollerus.

Taszycki wrote several reviews of works written by other experts in Sorbian studies. Some of these reviews are quite critical. Taszycki was also interested in Sorbian culture and literature. In particular, he dealt with the reception of Sienkiewicz's works among the Sorbs and published an annotated edition of letters by Jan Arnošt Smoler and Michał Hórnik to Kraszewski. In addition, as a university lecturer, he gave several lectures on Sorbian and the Sorbs. His knowledge of Sorbian has also been incorporated into his general onomastic works. 